\title{
Fraud in scientific research
}

Fraud in scientific research has been present for a long time. However, following the recent news that important healthcare authorities in Germany were charged with plagiarism, this issue has gained much attention.

At present, competent authorities are dealing with this problem with a more rigorous approach.

Thus, the number of cases of plagiarism, falsification, and even fabrication (invention) of results reported in scientific studies has been increasing over the last few years, suggesting that a "fraud epidemic" is spreading through the scientific community.

The current access to the internet and computer programs facilitates easier practice and detection of frauds.

In the United States, the Office of Research Integrity (ORI), Department of Health, of the United States Government monitors instances of fraud committed by scientists and is responsible for investigating these cases.

There have been cases of spontaneous retraction as well as cases wherein guilt is formally confirmed.

Such investigations are usually based on the Web of Science database, which is a digital library that catalogs articles published in top international scientific journals, or on studies available in the PubMed database.

The number of and reasons for the retraction of 2,047 studies from the PubMed database until May 2012 are as follows: 698 cases of fraud; 192 cases of suspected fraud; 200 cases of plagiarism; 437 cases involving scientific mistakes; 290 cases of duplicate publication; 108 cases involving other reasons; 182 cases involving unknown reasons.

In Brazil, the Conselho Nacional de Desenvolvimento Científico e Tecnológico (National Council of Scientific and Technological Development) - $\mathrm{CNPq}$ - is responsible for monitoring this issue.

The Fundação de Amparo à Pesquisa do Estado de São Paulo (Amparo Foundation for Research, State of São Paulo) FAPESP - released the "Code of Scientific Good Practices," which stipulates the fundamental principles of scientific integrity, and established rules and deadlines for investigating the possible cases of misconduct.

Since the code was established, the FAPESP has initiated 14 administrative proceedings for scientific misconduct, including 3 for falsification of research data, 1 for plagiarism associated with falsification of data, 1 for falsification of curricular data, and 1 for violation of ad hoc advisory confidentiality.

Due to the apparently increasing proportion of such cases, it is essential to spread awareness and knowledge about this problem within the scientific community to denounce such conduct and preserve scientific integrity.

\section{Rolf Gemperli}

Associate Editor 